\title{
Asymptomatic Plasmodium Parasites among Adults in Eastern Uganda: A Case of Donor Blood Screening at Mbale Regional Blood Bank
}

\author{
Simon Peter Inyimai, ${ }^{1,2}$ Mosses Ocan, ${ }^{2}$ Benjamin Wabwire, ${ }^{1}$ and Peter Olupot-Olupot $\mathbb{D}^{3,4}$ \\ ${ }^{1}$ Mbale Regional Blood Bank, Uganda \\ ${ }^{2}$ Mbarara University of Science and Technology, Uganda \\ ${ }^{3}$ Mbale Clinical Research Institute, P.O. Box 1966, Mbale, Uganda \\ ${ }^{4}$ Busitema University, Faculty of Health Sciences, Mbale Campus, P.O. Box 1460, Mbale, Uganda \\ Correspondence should be addressed to Peter Olupot-Olupot; polupotolupot@yahoo.com
}

Received 20 February 2018; Accepted 25 June 2018; Published 9 July 2018

Academic Editor: Sukla Biswas

Copyright (C) 2018 Simon Peter Inyimai et al. This is an open access article distributed under the Creative Commons Attribution License, which permits unrestricted use, distribution, and reproduction in any medium, provided the original work is properly cited.

\begin{abstract}
Background. There is a paucity of data on asymptomatic carriage of Plasmodium parasite among adult population in Eastern Uganda, an area of perennial high transmission of malaria. In this study, we estimated the prevalence of Plasmodium parasites in donor blood units at Mbale Regional Blood Bank (Mbale RBB), a satellite centre of the Uganda Blood Transfusion Service (UBTS). Method. This was a cross-sectional descriptive study in which 380 screened donor blood units were examined for the presence of Plasmodium parasites. A systematic random sampling technique using the interval of 7 was used in selecting the screened blood units for testing. Two experienced malaria slide microscopists ( $\mathrm{MC1}$ and $\mathrm{MC} 2)$ independently examined each thick and thin blood slide under high power magnification of X400 and then X1000 as stated on the study standard operation procedure (SOP). Each slide was examined for 100 oil immersion fields before the examiner declared them negative for Plasmodium parasites. The results by each microscopist's examination were tallied separately, and finally, the two tallies were compared. The third independent microscopist (MC3) was blinded to the results from $\mathrm{MC1}$ and $\mathrm{MC2}$, but whose role was to perform quality control on the slides randomly sampled and read $38(10 \%)$ of all the slides and was available to examine any slides with inconsistent findings by $\mathrm{MC1}$ or MC2. Results. All the microscopists were unanimous in all the slide readings. Five of the thick smears (1.3\%) confirmed the presence of Plasmodium parasites among donor blood units. Of these, 4/5 were from male donors. Plasmodium falciparum was identified in 4 positive samples, while Plasmodium malariae was identified in one of the donor units. Conclusion. The $1.3 \%$ prevalence of Plasmodium malaria parasites in screened donor blood units represents risk of malaria blood transfusion transmitted infection and a pool of community transmittable malaria infections, respectively.
\end{abstract}

\section{Background}

Globally, there has been a substantial reduction in malaria incidence as a result of scaling up of control and prevention efforts. There are currently renewed interests on initiatives for malaria elimination $[1,2]$ and accurate mapping of areas with malaria parasite prevalence [3]. This is especially in this era of malaria epidemiological transition in which some countries in Africa have documented either no decline [4], an increase in hospitalisations with severe Plasmodium falciparum (P. falciparum) malaria during the same period [5], or a resurgence of severe malaria following a period of sustained control [6]. Worldwide P. falciparum malaria causes 300-500 million clinical episodes, with up to 445,000 direct deaths attributed to the disease in 2017 [7]. In addition, malaria is a major coinfection to other diseases contributing to an estimated 3 million deaths annually across the world [8]. Ninety percent of these deaths occur in African children $<5$ years old $[9,10]$. Fifteen sub-Sahara African (SSA) countries account for $80 \%$ of the global malaria cases [7].

Control and/or prevention of malaria using vaccines has so far proven to offer limited short-term protection [7]. Thus, single-phased prevention strategies alone in many parts of Africa will not adequately contribute to substantial reduction 
in the disease burden. Strategies to limit malaria transmission in the community by breaking the malaria cycle and disrupting any further transmission have provided promising outcomes in malaria control and elimination efforts in malaria endemic areas [11]. However, these efforts require a combination of approaches and not a single intervention at a time [12]. Use of the once effective indoor residual spraying with Dichlorodiphenyltrichloroethane (DDT) in some endemic countries has been discouraged because of its potential health and environmental hazards [13], while in other settings, growing resistance to DDT has been reported [12]. Malaria control and elimination efforts need to be expanded to other previously recognised but poorly described risk of malaria transmission in communities, for instance, transmission of malaria through blood transfusion, especially in malaria endemic regions of the sub-Saharan Africa [3]. Targeting malaria transfusion transmitted infections will enhance the gains being reported from the current malaria control and elimination efforts [7]. Therefore, understanding the prevalence of both symptomatic and asymptomatic malaria would help inform targets for control and prevention efforts geared towards elimination of the disease. In Eastern Uganda available data demonstrates that spleen rates among children $<5$ years were high [14], and malaria transmission is intense $[14,15]$. There are no data on asymptomatic malaria carriage in adults in Eastern Uganda, just as in the rest of the country. Therefore, descriptions of human populations that harbour malaria parasites are critical. There are some studies evaluating use of chemotherapy for community control of malaria [16]. Other interventions for possible eradication of malaria parasitaemia in the community are being evaluated. The effectiveness of such interventions would be well informed by the understanding of the pools of asymptomatic carriage of malaria parasites in the community. Robust surveillance for asymptomatic carriage of malaria parasites, however, is largely lacking. Exploration of proxy estimation methods could yield useful data for mapping communities at risk or those that harbour potentially transmissible Plasmodia. We explored the potential of using Mbale RBB in Mbale municipality with a catchment of 27 districts in the region, as a hub for surveillance for asymptomatic carriage of Plasmodia. The facility receives blood units from two categories of voluntary donors; the "walk-in-donors" who voluntarily come to the regional facility to donate, and "out-reach-donors" who are accessed by the Mbale RBB mobile blood collection teams for blood donation in the communities in the catchment area including students. The blood collection teams are trained in counseling and selection of low risk blood donors in the community. Only healthy asymptomatic donors are recruited and these therefore comprise the right target population for surveillance for asymptomatic Plasmodia carriage. Before blood is considered safe and issued for transfusion, tests for blood group, viruses (HIV, Hepatitis B, and Hepatitis C), and syphilis (TPHA) are done. We have previously used data on viral screening from Mbale RBB to describe epidemiology of Hepatitis B and Hepatitis C in the communities [17]. Tests for haemoparasites such as Plasmodium are not routinely done; therefore, there is no data to describe asymptomatic malaria using donor blood in Eastern Uganda. This study was therefore designed to investigate the feasibility of surveillance for asymptomatic malaria in donor blood as proxy to estimation of community asymptomatic carriage of malaria in a malaria perennial high transmission Eastern Uganda.

\section{Materials and Methods}

This was a cross-sectional study conducted from 1 June to 31 July 2015 in Eastern Uganda. Screened donor blood units from Mbale RBB were examined for the presence of malaria parasites. The region has a stable high transmission burden for malaria with over 100 infective mosquito bites per person per year [15]. The Mbale RBB handles more than 30000 voluntary blood donations per annum.

2.1. Sample Size Calculation. Using the Kish, L. (1965) Survey Sampling; John Wiley and Sons, Inc. New York formulae, a sample size of 380-donor blood units was included in the study. The blood donor units that met the selection criteria were serially arranged. The blood units selected for testing were determined by referring to the Mbale RBB database. The database showed that out of the 30000 collected each year, $90 \%$ (27000) of them would be safe for blood transfusion.

2.2. Sampling Criteria. The study was conducted over 2 months for which a conservative blood collection forecast (monthly minimum 1250-maximum 2750 blood units) was made arriving at a study population of 2530 blood units. The study population of 2530 blood units was divided by the sample size of 380 , obtaining a sampling interval of 7 . The first unit to be selected was determined by putting pieces of paper containing numbers of the first ten units in a box. One paper piece was then picked at random and the number contained therein was considered as the first number. Every seventh unit, beginning from the currently selected blood unit number, was considered for the assay. Blood samples were extracted from the integral segment of the donor bag for analysis. All blood donor units that showed any form of hemolysis were excluded.

2.3. Data Collection. The demographic information of the blood donors without their personal identification information was collected using a data abstraction tool. From each selected blood donor unit, at the integral tubing, about $100 \mu \mathrm{l}$ of blood was collected into a cryovial with the help of a tube sealer. Thick and thin blood smears were made for each blood unit for identification and typing of Plasmodia parasites, respectively. The thick blood smears were stained with $10 \%$ Giemsa at PH 7.2, a stable methanol based Romanowsky stain. The stained blood smears were examined microscopically under X400 and X1000 objectives. The presence of Plasmodia parasites was noted based on their staining features with Giemsa stain. We further quantified the malaria parasites by examining microscopically the X100 objective. The number of Plasmodium parasites in the positive smears was counted against $500 \mathrm{WBC}$ and the value that was obtained was multiplied by 16 in order to obtain the Plasmodium parasitaemia level per microlitre of blood. We identified the Plasmodia species using thin smears. The positive smears 


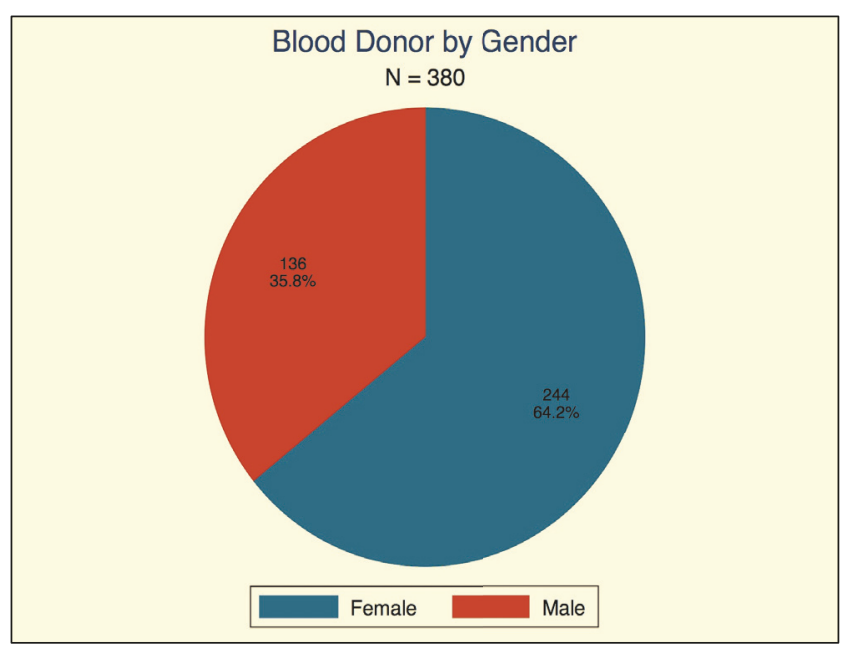

FIGURE 1: A pie chart of blood donors by gender.

were given arbitrary alphabetical letters A, B, C D, and E for confidentiality. Thin blood smears were stained with $10 \%$ Giemsa at PH 7.2, a stable methanol based Romanowsky stain and examined as described above.

2.4. Quality Control. The quality of results was ensured by staining freshly prepared Giemsa stain. The standard operating procedures of staining were observed in order to obtain consistent quality results. Two laboratory technologists read each pair of thin and thick slide preparation independently. The study SOP provided for a third independent microscopist whose duty was to ensure quality control by randomly sampling and reading 38 (10\%) of all the slides and to examine any slides with inconsistent findings by any of the first two microscopists.

2.5. Data Management and Analysis. The collected data was entered into Excel spread sheet 2007. Data was then transferred to SPSS ver 20.0 for analysis. Proportions were generated from the datasets.

2.6. Ethical Approval. The study was reviewed and approved by the Mbarara University of Science and Technology, Faculty of Medicine Research Ethics Committee (FRC), permission to conduct the study was granted by the Principal Medical Officer of Mbale RBB, and further ethical review and approval was done by the Mbale Regional Referral Hospital Research and Ethics Committee (MRRH-REC).

\section{Results}

Three hundred and eighty thick and thin blood films were made. A majority of the blood donors were males (Figure 1).

The mean age of the blood donors was $21 \pm 4$ years, range 18 - 22 years (Table 1 ).

3.1. The Distribution of Samples from Each District in the Catchment Area. The donor blood units sampled were from more than half (out of the total 27 districts) of the districts
TABLE 1: The age ranges of the blood donors.

\begin{tabular}{lc}
\hline Age Group & Number (\%) \\
\hline $18-22$ & $282(74.2)$ \\
$23-27$ & $66(17.4)$ \\
$28-32$ & $22(5.8)$ \\
$33-37$ & $07(1.8)$ \\
$38-42$ & $02(0.5)$ \\
$43-47$ & $01(0.3)$ \\
Total & $380(100.0)$ \\
\hline
\end{tabular}

that are covered by Mbale RBB collection teams. Generally, there was relative uniformity in the number of blood units. Nonetheless, a slightly larger proportion of the blood units were collected from donors within Mbale district; 42 (11.1\%) units were followed by Kumi district with 37 (9.74\%) units. The rest of the distribution is reflected in Figure 2.

The state of Plasmodium parasite parasitaemia among the blood units studied was summarized in Table 2 .

3.2. Prevalence of Plasmodium Parasites in the Donor Blood Units. Of the 380 donor blood units screened, 5/380 (1.3\%) carried Plasmodium parasites. Of the 5 donor blood units that had Plasmodium parasites, a majority (4/5) had Plasmodium falciparum, while only one had Plasmodium malariae parasites. Of the 136 donor blood units from female donors, 01 $(0.79 \%)$ had Plasmodium parasites while of the 244-donor blood units from male donors 04 (1.6\%) had Plasmodia parasites (Table 2).

\section{Discussion}

Our results indicate that the prevalence of Plasmodium parasites among ready-to-transfuse blood units was $1.3 \%$. This was lower than expected in this malaria high transmission region. This is epidemiologically and immunologically plausible, especially given the fact that individuals who had potentially acquired immunity to the disease may have been sampled. Furthermore, the screening criteria used to identify potential blood donors may have been very stringent that it eliminated a large proportion of asymptomatic carriers in the community. Nonetheless, the $1.3 \%$ represents a malaria pool with double the chance of transmitting the disease at the time of donation in the community, that is, through mosquito bites, and in the hospital through blood transfusion. In comparison, our findings are far below the immunological assays for similar surveys. For instance, the malaria antibody prevalence of $7.6 \%$ in Saudi Arabia suggests a possible lower diagnostic accuracy of blood slide for malaria compared to immunological assays, lower endemicity notwithstanding. But in West Africa, in malaria endemic setting, malaria in donor blood was 7\% [18] and 21\% [19], despite similarity in age of donors at these and out sites. This could be due to the differences in blood donor screening practices on one hand but conversely may be due to the differences in malaria seasons or immunity in the donors.

As expected for this region, the dominant species was $P$. falciparum malaria with $4 / 5$ positive samples confirming 


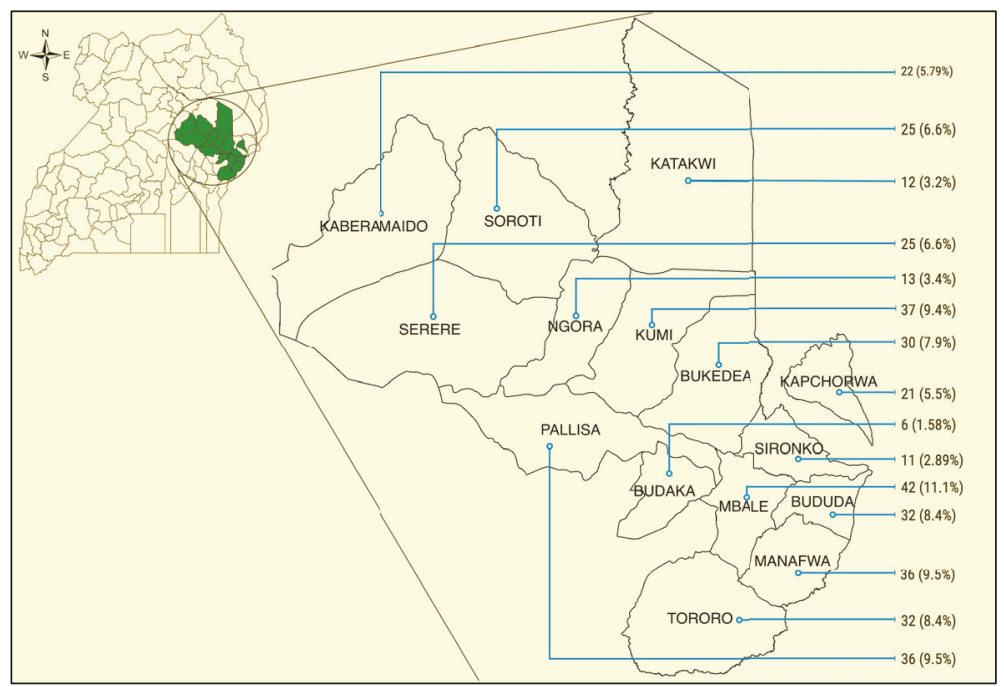

FIGURE 2: Infographic map of Uganda with insert showing the distribution of blood units sampled for the study.

TABLE 2: Blood group, malaria infection and asymptomatic parasite count.

\begin{tabular}{|c|c|c|c|c|c|}
\hline Case & Age group & Gender & Blood group & $\begin{array}{c}\text { Number of } \\
\text { Plasmodium } \\
\text { parasites/ } \mu \text { l of blood }\end{array}$ & $\begin{array}{c}\text { Plasmodium } \\
\text { species }\end{array}$ \\
\hline Case 1 & $18-23$ & MALE & $\mathrm{O}+$ & 48 & P. falciparum \\
\hline Case 2 & $18-23$ & FEMALE & $\mathrm{O}+$ & 62 & P. falciparum \\
\hline Case 3 & $24-30$ & MALE & $\mathrm{O}+$ & 48 & P. malariae \\
\hline Case 4 & $18-23$ & MALE & $\mathrm{O}-$ & 80 & P. falciparum \\
\hline Case 5 & $18-23$ & MALE & $\mathrm{AB}+$ & 32 & P. falciparum \\
\hline
\end{tabular}

positivity to the speciation. This is consistent with other series in Africa [20]. The role of asymptomatic parasitaemia in the transmission of malaria and whether or not it requires treatment remains poorly understood. We found low parasitaemia, probably noninfectious [21], but how long these parasites survive in immune human beings, reproduce, and eventually get to transmittable threat holds requires additional studies. Our thinking is that, all asymptomatic parasitaemia is a potential source of infection in the community.

In our study we also found $P$. malariae a rare malaria parasite in Uganda. Furthermore, because of its long incubation period and less severe clinical features, it is likely that the carriers of $P$. malariae infection do not show up for medication early, or if they do they do not receive appropriate medication for this parasite. Consequently, it continues to multiply in the body with a potential, overtime, to cause significant prevalence and/or coinfections with falciparum. We found that blood group $\mathrm{O}$ accounted for $4 / 5$ cases of asymptomatic malaria (Table 2). This is consistent with another series that indicated a strong association between blood $\mathrm{ABO}$ group with asymptomatic malaria $P=0.022$ and a high rate of parasitaemia in blood group $\mathrm{O} ; \mathrm{P}=0.003$ [22]. In our study, we did not look at other factors, but elsewhere, in similar malaria transmission settings, thrombocytopaenia $\left(<150 \times 10^{9}\right)$ was associated with asymptomatic malaria among blood donors [19]. The implication of our results with regard to blood transfusion and malaria control is potentially significant because asymptomatic malaria carries the risk of transmitting malaria parasites to the recipients of these blood units, of whom the majority are pregnant mothers and immunologically naive children. Our observations have demonstrated Plasmodia in screened donor blood. We recommend better and robust ways of screening for malaria in donor blood to protect blood recipients from these infections [23]. Programmes that target asymptomatic malaria pool should be included in community malaria control programs.

\section{Conclusion}

Malaria causing parasites were present in $1.3 \%$ of the donor blood units. This is of clinical and public health significance since it represents a risk of community transmission of malaria from blood transfusion, and also through mosquito bites. Use of more efficient, highly sensitive, and specific diagnostics techniques would improve the accuracy of asymptomatic malaria surveillance using donor blood.

\section{Data Availability}

The data used to support the findings of this study are available from the corresponding author upon request. 


\section{Ethical Approval}

The authors conducted their research within the provisions of ethical standards in Uganda. The Mbale Regional Referral Hospital Research \& Ethics Committee (MRRH-REC) approved the study and local permission to conduct the study was obtained from Mbale RBB.

\section{Disclosure}

The Mbale Clinical Research Institute (MCRI, http://www .mcri.ac.ug/), a research entity affiliated to the Uganda National Health Research Organization (UNHRO), permits the publication of this manuscript.

\section{Conflicts of Interest}

The authors declare no conflicts of interest.

\section{Authors' Contributions}

Simon Peter Inyimai, Mosses Ocan, and Benjamin Wabwire participated in various stages of data collection and participated in writing of the manuscript; Peter Olupot-Olupot conceived the idea and wrote the manuscript. All authors approved the final manuscript.

\section{Acknowledgments}

The authors acknowledge Mbale Regional Blood Transfusion Service, Mbale Clinical Research Institute, and all the Mbale RBB outreach staff for their various contributions to this work. They thank Gideon Nyutu for the graphic work.

\section{References}

[1] IS Global, "Malaria elimination initiative," 2018, https://www .isglobal.org/en/-/malaria-elimination.

[2] A. A. Lover, K. E. Harvard, A. E. Lindawson et al., "Regional initiatives for malaria elimination: Building and maintaining partnerships," PLoS Medicine, vol. 14, no. 10, Article ID e1002401, 2017.

[3] R. W. Snow, B. Sartorius, D. Kyalo et al., "The prevalence of Plasmodium falciparum in sub-Saharan Africa since 1900," Nature, vol. 550, no. 7677, pp. 515-518, 2017.

[4] A. Roca-Feltrer, I. Carneiro, and J. R. M. Armstrong Schellenberg, "Estimates of the burden of malaria morbidity in Africa in children under the age of 5 years," Tropical Medicine \& International Health, vol. 13, no. 6, pp. 771-783, 2008.

[5] E. A. Okiro, D. Bitira, G. Mbabazi et al., "Increasing malaria hospital admissions in Uganda between 1999 and 2009," BMC Medicine, vol. 9, article 37, 2011.

[6] M. J. Hamel, K. Adazu, D. Obor et al., "A reversal in reductions of child mortality in Western Kenya, 2003-2009," The American Journal of Tropical Medicine and Hygiene, vol. 85, no. 4, pp. 597605, 2011.

[7] WHO, "World malaria report 2017," Tech. Rep., World Health Organization, 2017, http://www.who.int/malaria/publications/ world-malaria-report-2017/report/en/.
[8] N. White et al., "WHO, the Global Fund, and medical malpractice in malaria treatment," Lancet, vol. 363, no. 9415, p. 1160, 2004.

[9] J. Cook, D. Hergott, W. Phiri et al., "Trends in parasite prevalence following 13 years of malaria interventions on Bioko island, Equatorial Guinea: 2004-2016," Malaria Journal, vol. 17, no. $1,2018$.

[10] S. Githinji, R. Oyando, J. Malinga et al., "Completeness of malaria indicator data reporting via the District Health Information Software 2 in Kenya, 2011-2015," Malaria Journal, vol. 16, no. 1, 2017.

[11] D. M. Parker, J. Landier, A. M. Thu, K. M. Lwin, G. Delmas, and F. H. Nosten, "Scale up of a Plasmodium falciparum elimination program and surveillance system in Kayin State, Myanmar," Wellcome Open Research, vol. 2, 2017.

[12] T. L. Russell, N. W. Beebe, R. D. Cooper, N. F. Lobo, and T. R. Burkot, "Successful malaria elimination strategies require interventions that target changing vector behaviours," Malaria Journal, vol. 12, no. 1, article 56, 2013.

[13] G. Chang, "Persistent organochlorine pesticides in aquatic environments and fishes in Taiwan and their risk assessment," Environmental Science and Pollution Research, vol. 25, no. 8, pp. 7699-7708, 2018.

[14] S. Kotlyar, J. Nteziyaremye, P. Olupot-Olupot, S. O. Akech, C. L. Moore, and K. Maitland, "Spleen volume and clinical disease manifestations of severe plasmodium falciparum malaria in African children," Transactions of the Royal Society of Tropical Medicine and Hygiene, vol. 108, no. 5, Article ID tru040, pp. 283289, 2014.

[15] M. Kilama, D. L. Smith, R. Hutchinson et al., "Estimating the annual entomological inoculation rate for Plasmodium falciparum transmitted by Anopheles gambiae s.l. using three sampling methods in three sites in Uganda," Malaria Journal, vol. 13, no. 1, article 111, 2014.

[16] T. Druetz et al., "Impact evaluation of seasonal malaria chemoprevention under routine program implementation: a quasiexperimental study in burkina faso," The American Journal of Tropical Medicine and Hygiene, vol. 98, no. 2, pp. 524-533, 2018.

[17] C. L. Cooper, E. Mills, B. O. Wabwire, N. Ford, and P. OlupotOlupot, "Chronic viral hepatitis may diminish the gains of HIV antiretroviral therapy in sub-Saharan Africa," International Journal of Infectious Diseases, vol. 13, no. 3, pp. 302-306, 2009.

[18] J. A. Faruk, G. O. Ogunrinde, and A. I. Mamman, "Observation of blood donor-recipient malaria parasitaemia patterns in a malaria endemic region," Journal of Tropical Medicine, vol. 2017, Article ID 7149261, pp. 1-5, 2017.

[19] C. Igbeneghu, A. B. Odaibo, and D. O. Olaleye, "Impact of asymptomatic malaria on some hematological parameters in the iwo community in southwestern nigeria," Medical Principles and Practice, vol. 20, no. 5, pp. 459-463, 2011.

[20] D. Ayala, G. L. Goff, V. Robert, P. De Jong, and W. Takken, "Population structure of the malaria vector Anopheles funestus (Diptera: Culicidae) in Madagascar and Comoros," Acta Tropica, vol. 97, no. 3, pp. 292-300, 2006.

[21] G. Covell, "Relationship between malarial parasitaemia and symptoms of the disease: a review of the literature," Bulletin of the World Health Organization, vol. 22, pp. 605-619, 1960.

[22] G. Alemu and M. Mama, "Assessing $\mathrm{ABO} / \mathrm{Rh}$ blood group frequency and association with asymptomatic malaria among blood donors attending arba minch blood bank, South Ethiopia," Malaria Research and Treatment, vol. 2016, Article ID 8043768, pp. 1-7, 2016. 
[23] E. K. Butler and J. McCullough, "Pathogen reduction combined with rapid diagnostic tests to reduce the risk of transfusiontransmitted infections in Uganda," Transfusion, vol. 58, no. 4, pp. 854-861, 2018. 


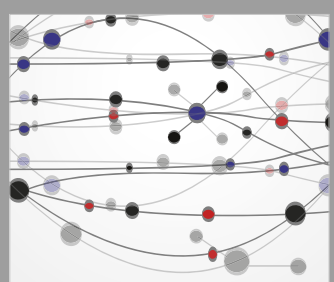

The Scientific World Journal
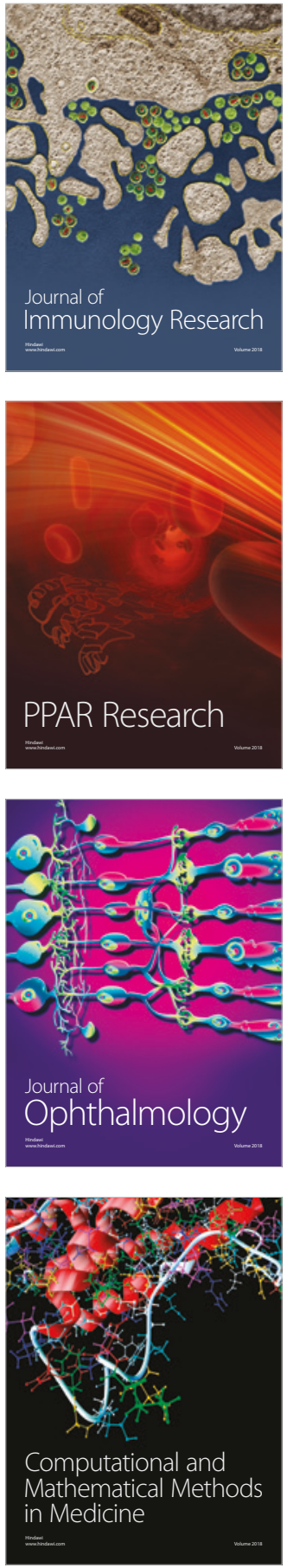

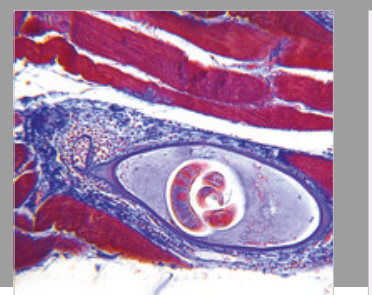

Gastroenterology Research and Practice

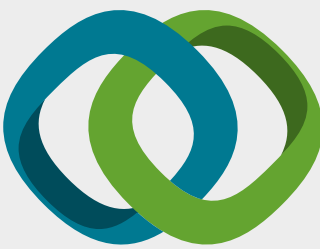

\section{Hindawi}

Submit your manuscripts at

www.hindawi.com
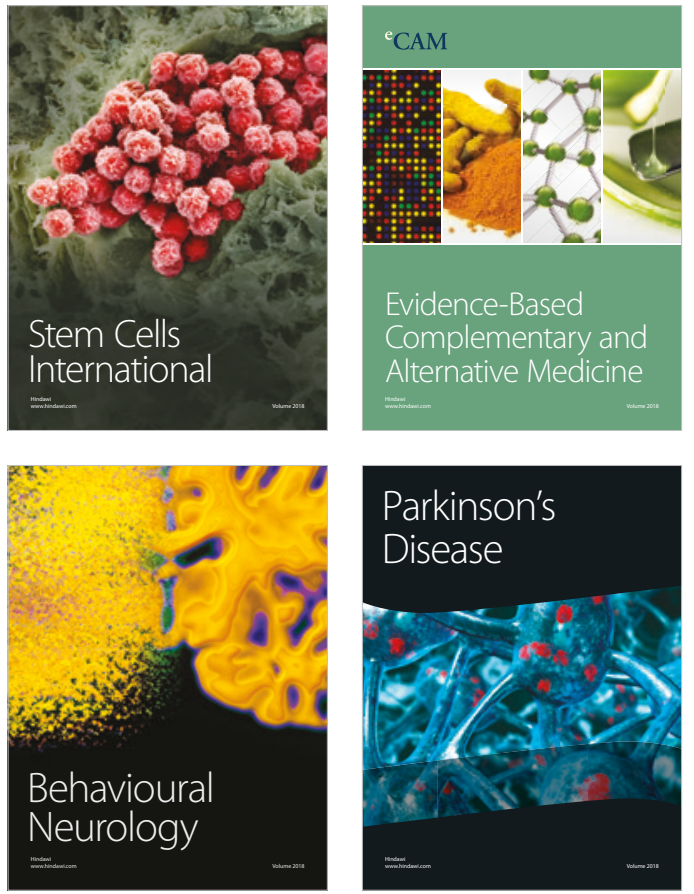

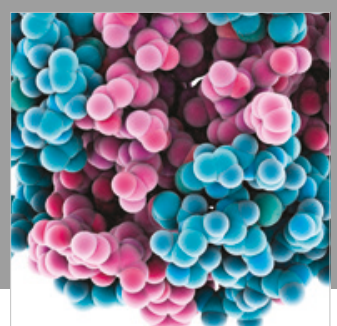

ournal of

Diabetes Research

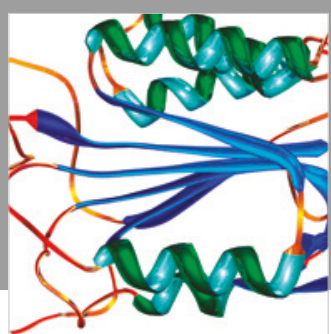

Disease Markers
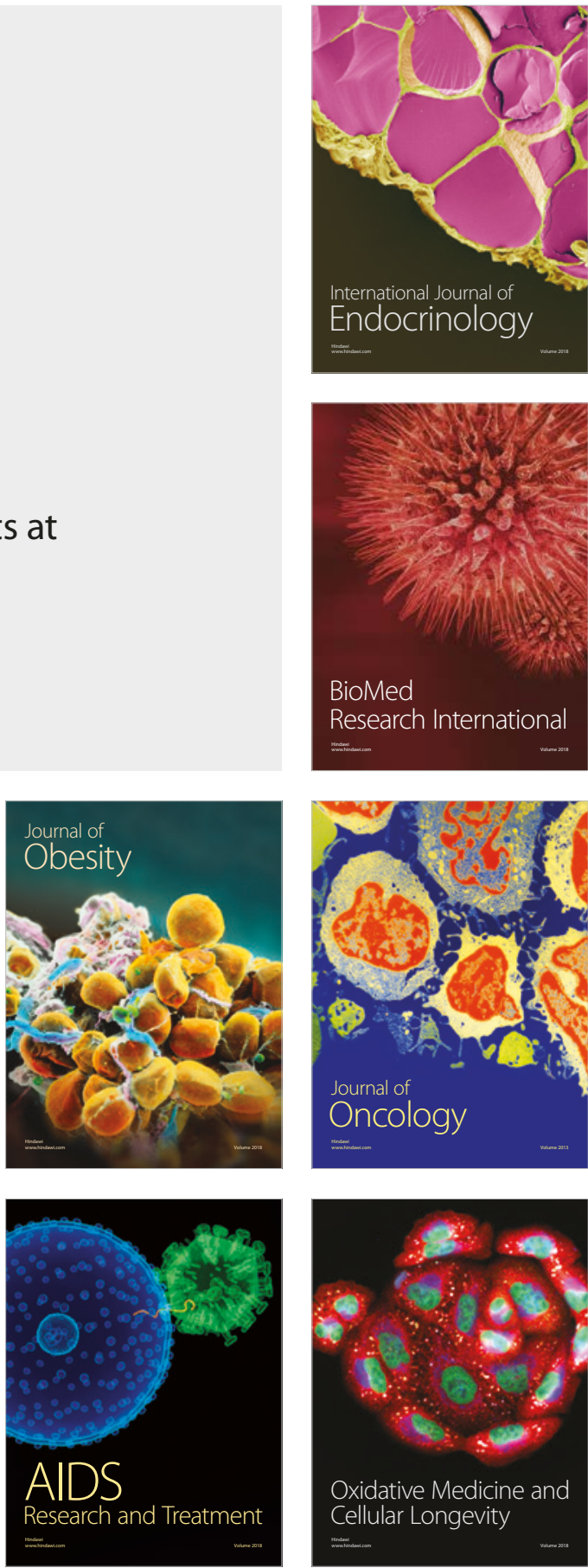